ÉGYPTE

monde arabe

\section{Égypte/Monde arabe}

4-5 | 2001

L'Égypte dans le siècle, 1901-2000

\title{
Rapport sur la crise économique et sociale
}

Mirrit Ghâli, Rapport sur la crise économique et sociale, Le Caire, août 1952 (sans éditeur), 60 pages

\section{Iman Farag}

\section{OpenEdition}

\section{Journals}

Édition électronique

URL : https://journals.openedition.org/ema/891

DOI : $10.4000 /$ ema.891

ISSN : 2090-7273

\section{Éditeur}

CEDEJ - Centre d'études et de documentation économiques juridiques et sociales

\section{Édition imprimée}

Date de publication : 30 juin 2001

Pagination : 319-322

ISBN : 2-87027-963-9

ISSN : $1110-5097$

\section{Référence électronique}

Iman Farag, «Rapport sur la crise économique et sociale », Égypte/Monde arabe [En ligne], 4-5 | 2001, mis en ligne le 08 juillet 2008, consulté le 07 juillet 2022. URL : http://journals.openedition.org/ema/ 891 ; DOI : https://doi.org/10.4000/ema.891

Ce document a été généré automatiquement le 7 juillet 2022

Tous droits réservés 


\section{Rapport sur la crise économique et sociale}

Mirrit Ghâli, Rapport sur la crise économique et sociale, Le Caire, août 1952 (sans éditeur), 60 pages

\section{Iman Farag}

1 L'ouvrage est paru en août 1952, un mois après la prise du pouvoir par les officiers libres. Tenter de percer le sens de l'anticipation de l'auteur ou le caractère prédicatif de l'ouvrage ne mène pas très loin. Pas plus qu'on ne peut savoir si ce dernier a voulu rattraper en vitesse l'événement politique qui marquait la fin de l'ancien régime en rédigeant ce rapport éclair à l'intention des nouveaux dirigeants de l'Égypte. En revanche, la date de parution est peut être bien ce qui fait du Rapport sur la crise un des livres oubliés. Et cela en dépit de la stature politique et intellectuelle de son auteur.

Qu'un Mirrit Ghâli, auteur par ailleurs d'un Projet de réforme agraire, s'intéresse à la crise économique et sociale, n'a rien d'incongru. Dans les années 1940, il est un des principaux fondateurs du Groupe de la renaissance nationale. Cercle élitiste, réformiste et a-politique sur la carte des mouvements intellectuels, le GRN occupe une place particulière. Dans l'historiographie ce serait la seule expression "authentiquement " libérale d'une ère dite libérale. D'un côté, une réflexion sur la pleine citoyenneté, conçue comme gigantesque projet pédagogique à l'échelle de la Nation, et dont le point d'ancrage et le laboratoire seraient l'appareil d'État : La réforme de l'outil gouvernemental (1945, 2 ${ }^{\circ}$ édition), en constituerait le programme. De l'autre, une évaluation, sinon une " mesure » de ce peu auquel les classes possédantes devaient renoncer si elles tenaient à préserver leurs énormes privilèges. Pour l'époque, cet euphémisme veut dire réforme agraire. Sauvetage du libéralisme donc, par la réforme du fonctionnement des institutions, mais aussi du minimum de preréquisits sociaux qui leur permettraient de fonctionner. Autrement dit, dès lors qu'est reconnue aux individus l'égalité des droits politiques, il importe de penser les dispositifs sociaux et pédagogiques, permettant de garantir une certaine cohérence à cette reconnaissance, et de ce point de vue, réforme agraire et accès à l'éducation vont de pair. 
3 Le Rapport sur la crise s'inscrit dans le droit fil de ces préoccupations : "J'ai omis à dessein de traiter des questions politiques, ne les abordant que lorsqu'elles avaient trait directement aux aspects économiques et sociaux et cela afin de limiter l'enquête aux faits incontestables. Également pour attirer l'attention sur les considérations économiques, souvent négligées par l'effet de l'enthousiasme politique. Il est normal que les questions politiques occupent la première place dans les préoccupations des hommes, qui ne vivent pas seulement de pain mais de valeurs suprêmes et de dignité. Cependant, l'aspect matériel aura vite fait de reprendre ses droits » (intro).

4 Le Rapport sur la crise est significatif en termes de construction "scientifique " d'une question sociale. Quelques éléments me semblent justifier une telle lecture, qui devraient être mis en regard avec les procédés de déchiffrement et d'argumentation entrepris par les réformistes.

5 D'abord, l'agencement des chiffres et des lettres si l'on peut dire: «Je n'ai pas voulu alourdir ces pages de tableaux et graphiques, provenant de sources officielles, afin d'en faciliter la lecture. J'espère rééditer le premier chapitre dans une version plus scientifique, comprenant références et statistiques. » De ce futur ouvrage, on n'a pas trace, et la notice figurant en deuxième de couverture ne permet pas d'en savoir plus sur le lecteur auquel est destiné ce « rapport » simplifié.

Cette crise, par rapport à quoi la mesurer ? Sur ce point, le Rapport semble se distinguer des usages en vigueur. Les contemporains de M. G. et ses prédécesseurs semblent en effet privilégier un standard unique puisque les retards de leur société ne prennent sens à leurs yeux que par rapport aux progrès de l'Occident (ou du Japon). Or, M. G. réfute explicitement une telle perspective. Ni comparaison avec le présent des sociétés européennes ou de sociétés comparables donc, ni «indices synthétiques »- et de ce point de vue on rejoint des questions actuelles - la seule mesure qui vaille, consiste à opposer le présent de l'Égypte à son passé, proche. «Il est normal qu'un peuple soit riche et un autre pauvre. Le premier n'est pas forcément plus heureux que le second (...). Ce qui nous importe est le sens du progrès et du retard économique. Si nous voulons juger de notre économie au présent, il faut la comparer à l'état antérieur de l'économie (...) pour savoir si l'Égypte va vers la richesse ou la pauvreté. Autrement dit, nous accepterions que l'Égypte d'aujourd'hui soit pauvre, si son évolution la conduisait vers le progrès » (p. 5).

7 Précisons pour lever les ambiguïtés que, pour M. G., retracer l'historique de la crise présente n'est pas exactement considérer ce que seraient des «dynamiques de paupérisation ». Ce passé proche est notamment celui de l'établissement progressif de la souveraineté de l'État égyptien et de ce que cela impliquait en termes d'accroissement des dépenses publiques, que ne contrebalançait pas un accroissement parallèle des ressources. À croire que l'économie du pays au tout début du XX' siècle aurait été plus proche d'une "économie naturelle», fonctionnant sans entraves étatiques; "relativement équilibrée, non parce que saine, mais parce que les besoins exprimés étaient moindres et l'effet des courants politiques et sociaux moins déterminants » (p. 7). À ce niveau de réflexion rétrospective relative à l'État, s'adjoint un autre niveau - on reviendra sur leur articulation - qui concerne le social. L'argumentation sur la crise couvre ainsi un spectre qui va de la multiplication des représentations diplomatiques de l'Égypte à l'étranger aux consommations " populaires ». Sur ces dernières - variétés de cotonnades mais aussi sucre, thé, café, tabac, les plaisirs du pauvre en fait - M. G. n'ignore pas le double sens des estimations: 
la baisse de consommation témoigne bien de la crise, mais sa hausse pourrait être également signifier le report des besoins frustrés sur ce qui reste à portée des bourses vides.

Comment le savoir et sur quoi appuyer l'argument quand les chiffres viennent à manquer? C'est " aux souvenirs du passé enregistrés dans la mémoire » que fait appel M. G. Appréciations toutes subjectives, mobilisées faute de mieux, à des fins de rétrospective : «Quant aux autres composantes du niveau de vie, plus particulièrement le logement et l'habillement, il n'existe pas de données statistiques qui permettraient de les évaluer avec la même rigueur que la consommation alimentaire. On ne peut là que se baser sur la mémoire et les estimations subjectives, en dépit des écarts que cela peut susciter quant aux appréciations de l'évolution passée (...); sans faire dire à ces chiffres plus qu'ils ne peuvent dire, ils ne semblent indiquer aucune amélioration dans le niveau de l'habillement. La mémoire, quant à elle, enregistre que les gens des campagnes étaient bien mieux vêtus il y a 20 ans qu'ils ne le sont aujourd'hui » (p. 9).

Un élément qu'il semble important de relever est la structure même du Rapport, qui donne titre et agencement aux trois chapitres; aspects, causes et traitement de la crise. Autant dire symptômes, étiologie et traitement et, sur ce point, M. G. est bien de son temps et de l'universel réformiste, si l'on peut dire ainsi. Là où cela pose un problème, que l'élégance formelle de la démarche thérapeutique ne parvient pas à masquer, c'est que symptômes et étiologie ne sont en fait qu'une seule et même chose. Tant par ses aspects que par ses causes, la crise économique et sociale de l'Égypte est bien celle d'un accroissement insuffisant des ressources du pays au regard de l'accroissement de sa population, qui aura doublé depuis le début du siècle. C'est ce que dicte la rationalité économique (scientifique), et on comprend mieux la fonction de la mesure par rapport au passé. Est-ce à dire que la distribution de ces mêmes ressources soit une question subsidiaire ? Certes non, mais cela relève d'une « raison politique ».

10 Le Rapport est en fait balancement et va-et-vient entre ces deux ordres d'impératifs. Une vue sur la crise que ne partageaient pas alors les mouvements revendicatifs (communistes, ouvriers), plus préoccupés par la juste répartition des ressources que par leur accroissement. C'est une démarche inverse (et complémentaire ?) qu'a voulu suggérer M. G. en dépolitisant la « crise » : «Écrivains et journalistes qui traitent de nos problèmes économiques et sociaux, se limitent à la redistribution de la richesse et reprennent à l'envi les mêmes chiffres connus de tous; ils commentent indéfiniment l'écart entre riches et pauvres, représentent le riche vivant dans son palais et baignant dans le superflu, face au pauvre qui meurt faute du nécessaire, croyant démontrer que la justice redistributive sera en mesure de mettre fin à la pauvreté, l'ignorance et la maladie. Aux yeux du public, voire de certains éléments de l'élite et de quelques étrangers, ceci constituerait notre principal problème (...). Évoquer la distribution des ressources est de plus un outil facile pour des propagandes en tous genres (...) au point que les gens perdent leur capacité de jugement et voient en tout ce qui n'est pas la stricte égalité, une intolérable injustice. Du rejet des riches on va vers le rejet des représentants de l'ordre publique et des hommes politiques; ce qui à n'en pas douter représente un danger pour l'État » (p. 23).

11 Il est à peu près certain, sous réserve d'inventaire, que le traitement de la crise (chapitre III) que prône le Rapport ne dépareille en rien des idées qui constituaient le fond commun des réformistes : développement de l'agriculture et réforme agraire (de la propriété, la location et le travail agricole), dont l'enjeu est tout autant sociopolitique 
qu'économique puisqu'il s'agit de limiter progressivement la grande propriété et d'accroître en parallèle les exploitations moyennes viables par la taille ; aide de l'État au secteur industriel privé sans protectionnisme outrancier; meilleure allocation des ressources nationales (consommation, impôts, épargne); contrôle des naissances, encouragement de l'émigration vers l'étranger et enfin réforme des administrations.

12 C'est en revanche par la construction des arguments, par le souci didactique tout autant que celui de " dépassionner la crise » (qui se prête généralement aux passions), que le Rapport - qui porte bien son nom - mérite l'attention. Le premier chapitre présente les aspects de la crise vus à travers le niveau de vie, le budget de l'État et la balance commerciale, le tout assorti de définitions. L'intégralité de ces aspects est ramenée aux causes de la crise, sinon sa cause unique; l'insuffisant accroissement des ressources au regard de l'augmentation de la population. Le deuxième chapitre superfétatoire ou presque - réitère ce que l'on savait déjà : inadéquation entre les ressources et la population. À cela, et au nombre des causes,

13 M. G. rajoute la mauvaise répartition des ressources, essentiellement la propriété agricole (et qu'en toute logique, on pourrait compter comme aspect et non seulement cause de la crise), il se rattrape en soulignant combien est centrale la faiblesse des ressources. Causes subsidiaires enfin, l'incohérence des politiques menées depuis l'accès de l'Égypte à sa souveraineté (1923), les surenchères partisanes et la faible performance des appareils d'État.

14 Enfin, l'ultime mise à jour se laisse entrevoir dans ces mots: "J'ai écrit les lignes précédentes avant le dernier "mouvement béni" et n'ai voulu ni les supprimer ni en modifier le contenu, même si cela ressemble à un plaidoyer prononcé après l'obtention d'un jugement favorable » (p. 34).

INDEX

Mots-clés : révolution de 1952, crise sociale

\section{AUTEUR}

IMAN FARAG

Cedej 